\title{
Structural peculiarities associated with multiflagellate sperm in the emu,
}

\section{Dromaius novaehollandiae}

\author{
Lizette du Plessis ${ }^{1}$ and John T. Soley ${ }^{2}$
}

Short title: Multiflagellate sperm in the emu

Address: ${ }^{1}$ Electron Microscope Unit, Department of Anatomy and Physiology, Faculty of Veterinary Science, University of Pretoria, Onderstepoort 0110, South Africa

${ }^{2}$ Department of Anatomy and Physiology, Faculty of Veterinary Science, University of Pretoria, Onderstepoort 0110 , South Africa

Corresponding author: Lizette du Plessis

e-mail:lizette.duplessis@up.ac.za

\begin{abstract}
Multiflagellate sperm represent a rare defect in mammals and also in the emu where an incidence of only $1 \%$ multiflagellate sperm was recorded in semen samples from 15 birds. Biflagellate sperm were the most frequent form of the defect observed, but 3- to 5-tailed sperm were also noted. An association was apparent between multiple tails and macrocephalic sperm which accounted for $0.6 \%$ of multiflagellate sperm. Structural features of the defect were generally similar to those seen in mammals. The duplicated tails shared a single midpiece which housed supernumerary centriolar complexes, each surrounded by a mitochondrial sheath. A single row of mitochondria separated adjacent centriolar complexes. Elements of the connecting piece (segmented columns, capitellum) appeared normal. The nuclear base appeared flat, staggered or scalloped depending on the number
\end{abstract}


and depth of additional implantation fossae. Multiflagellate emu sperm of normal head dimensions displayed a widened nuclear base (in the form of an attenuated peripheral nuclear extension) to accommodate the attachment of the additional centriolar complexes. Defective mammalian sperm do not show this modification of the nuclear base as the inherently wider sperm head is able to accommodate the supernumerary centrioles. Although often spiraled around each other, the duplicated principal pieces of the tail were generally separated and free. However, in some cells the proximal parts were collectively bound within the plasmalemma. Multiflagellate sperm appear to have a dual origin with some defective cells originating from incomplete cytokinesis and others as a result of abnormal centriolar duplication.

Keywords: emu, Dromaius novaehollandiae, spermatozoa, multiple tails

\section{Introduction}

Significant advances have recently been made in gathering data relevant to the application of artificial insemination (AI) technology in the ratite industry [1-4]. However, considering the primary importance of semen evaluation in establishing AI programmes, relatively little information is currently available on the morphology of sperm defects in this commercially important group of birds, particularly at the ultrastructural level. Whereas various abnormal forms of spermatozoa have been named in the ostrich [5] and emu [6] but not described, and some morphological data provided on cytoplasmic droplets in ostrich sperm [7,8], the only comprehensive accounts of abnormal sperm structure in ratites are those on the emu [9-11]. The evaluation of sperm motility, sperm concentration and sperm morphology form the three most important parameters in semen analysis [12-16]. The importance of sperm morphology in the assessment of semen quality in ratites has been 
recognised by Bertschinger et al [5] who note that in the ostrich "sperm morphology is the single most important factor in predicting fertility".

The occurrence of multiflagellate sperm has been reported in a number of mammalian species including the stallion [17,18], bull [19,20], boar [21-23] and dog [24], as well as in man [25-30]. This anomaly is characterised by the presence of two or more flagella and is considered a rare condition which adversely influences sperm motility and therefore fertility [19,26,27]. It has also been reported that a high proportion of macrocephalic sperm often, but not always [21], display multiple flagella $[20,26,27,29,31]$. The ultrastructural features of this defect have been thoroughly described, demonstrating the uniformity of the anomaly across different mammalian species [19,26,27].

In contrast, little information has been presented on the occurrence, incidence and structure of multiflagellate sperm in birds. Most classification systems on avian sperm defects fail to reflect multior biflagellate sperm [32-36]. However, this anomaly has been mentioned [37-39], briefly described and/or illustrated in some avian species such as the Houbara bustard [40], goose [41-43], duck [44], guinea fowl [45] and, more recently, in the emu [9]. With the exception of a detailed study on the ultrastructure of multiple sperm tails in the drake [44], the morphological features of this defect are poorly understood in birds. This paper presents a comprehensive description of multiflagellate sperm in the emu utilising both light and electron microscopy and provides supporting information on the origin of the defect. 


\section{Materials and methods}

Semen and testes samples were collected during the breeding season from 15 sexually mature and active emus, Dromaius novaehollandiae, following slaughter at a commercial abattoir. Semen samples were gently expressed from the distal ductus deferens and immediately fixed in $2.5 \%$ glutaraldehyde in 0.13M Millonig's phosphate buffer, $\mathrm{pH}$ 7.4. Smears for light microscopy (LM) were prepared from the fixed semen samples and stained with Rapiddiff ${ }^{\circledR}$ (Clinical Sciences Diagnostics, Johannesburg, South Africa). The smears were examined with a Leica DM6000-B light microscope (Leica Microsystems, Wetzlar, Germany) using a 100x oil immersion objective and phase contrast illumination to evaluate sperm morphology. The incidence of multiflagellate sperm defects was determined by counting for each bird the number of multiple tailed sperm present in a total of 300 cells.

The glutaraldehyde-fixed semen samples were also routinely prepared for scanning (SEM) and transmission (TEM) electron microscopy using standard techniques as previously described [9]. For SEM, the semen samples were dehydrated through a graded ethanol series and incubated in hexamethyldisilazane (HMDS). A drop of this suspension was placed on a glass coverslip and allowed to air-dry before sputter-coating the samples with gold. The samples were viewed in a JEOL Model 6010LA scanning electron microscope (JEOL Electron Optics Instrumentation, Tokyo, Japan) operated at $5 \mathrm{kV}$.

Small blocks of testicular material were fixed in $4 \%$ glutaraldehyde in $0.13 \mathrm{M}$ Millonig's phosphate buffer, $\mathrm{pH} 7.4$ and routinely prepared for TEM. Thin sections of both concentrated sperm and testicular material were stained with lead citrate and uranyl acetate and viewed in a Philips CM10 
transmission electron microscope (Philips Electron Optical Division, Eindhoven, The Netherlands) operated at $80 \mathrm{kV}$.

To distinguish between sperm of normal dimensions and macrocephalic sperm, linear measurements of head (nuclear) length and tail length $(\mathrm{n}=30)$ were taken on light micrographs, and nuclear base width measurements $(n=25)$ on transmission electron micrographs. Measurements were processed using the Soft Imaging System iTEM software (Olympus, Münster, Germany) and expressed as the average \pm s.d.

\section{Results}

\section{Light microscopy}

Multiflagellate sperm were readily identified by light microscopy (Fig. 1) and were present in all the samples, albeit in low numbers. Sperm displaying multiple tails formed only $1 \%$ of the total cell count, although the anomaly accounted for $7 \%$ of total sperm defects. Multiple-tailed sperm were often, but not always, associated with macrocephalic sperm. Abnormal cells displaying this association formed $0.6 \%$ of the total cell count while sperm with normal sized heads and multiple tails comprised $0.4 \%$ of the total cell count. The ratio of macrocephalic sperm with multiple flagella to multiflagellate sperm with normal heads was 3:2. Macrocephalic sperm with a single flagellum were also observed and constituted $1.5 \%$ of the total cell count. The heads of macrocephalic sperm measured approximately 1.5 times the length of normal sperm heads and exhibited a wider head base (Fig. 1b). The difference in head base width was also 1.5 times as determined from transmission electron micrographs. The individual tails (midpiece, principal piece and endpiece) of multiflagellate sperm were of similar length $(52.7 \pm 3.09 \mu \mathrm{m} ; \mathrm{n}=15)$, irrespective of head size and conformed to 

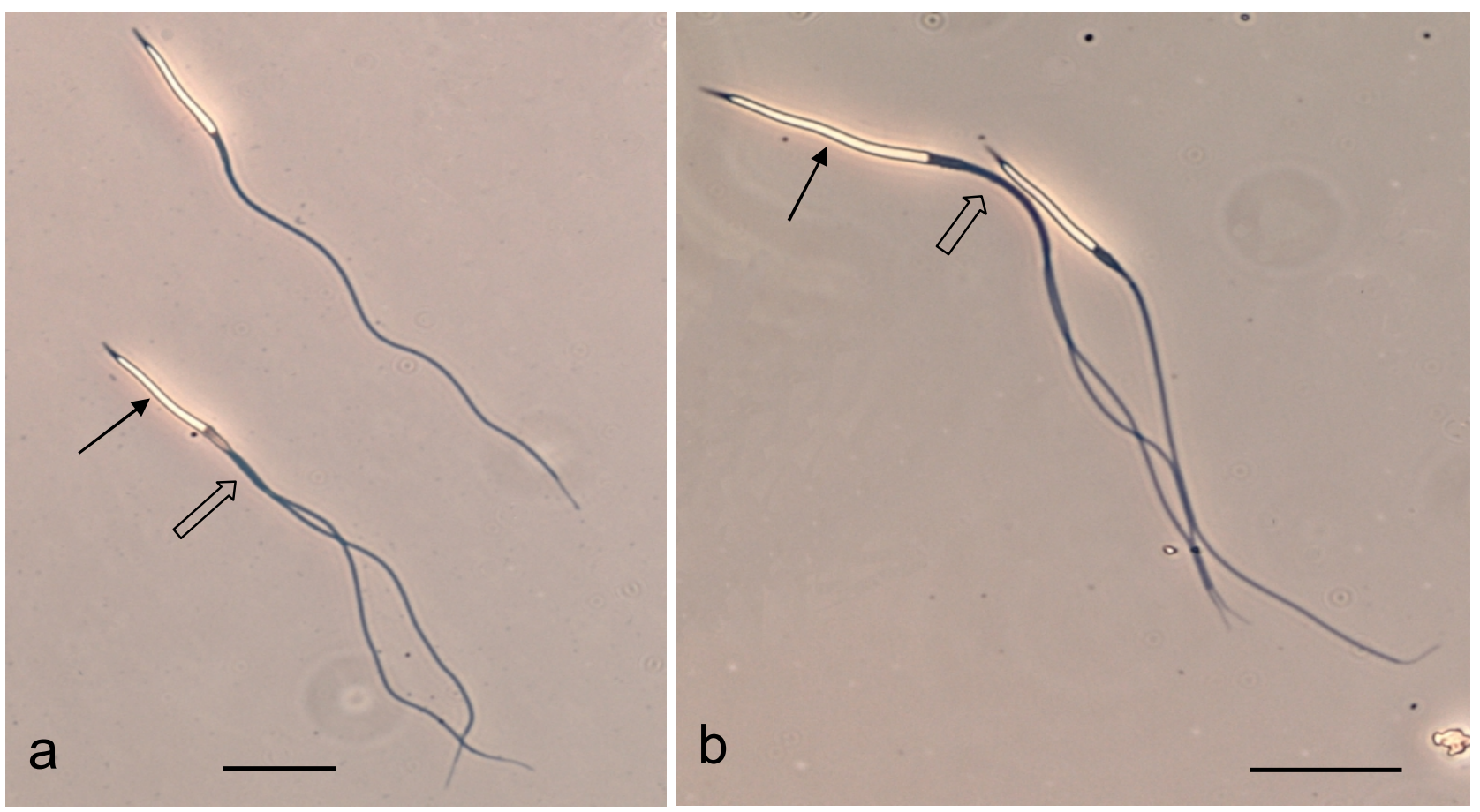

Fig. 1. Light micrographs showing (a) a normal sized sperm head (solid arrow) with a double tail (open arrow) and (b) a macrocephalic sperm (solid arrow) with a double tail (open arrow). Note the single midpiece and apparently fused proximal principal pieces of both biflagellate sperm. Bar $=10 \mu \mathrm{m}$.

the tail length of normal sperm $(53.11 \pm 3.07 \mu \mathrm{m} ; \mathrm{n}=15)$. On LM a maximum of five tails were observed on individual spermatozoa, although the biflagellate form of the defect was most commonly encountered. Sperm displaying this anomaly exhibited a single midpiece. The proximal aspect of the multiple principal pieces appeared fused or intimately spiralled with the greater portion of the duplicate tails lying free (Figs. 1a,b).

\section{Electron microscopy}

Scanning electron microscopy generally confirmed the light microscopic features of the anomaly. In most multiflagellate sperm a single but widened midpiece was evident (Fig. 2), although in some instances biflagellate sperm appeared to possess a double (split) midpiece. The proximal part of the 
individual principal pieces of the multiflagellate tails were either spiralled around each other (Fig. 2a) or lay closely aligned (Fig. 2b).
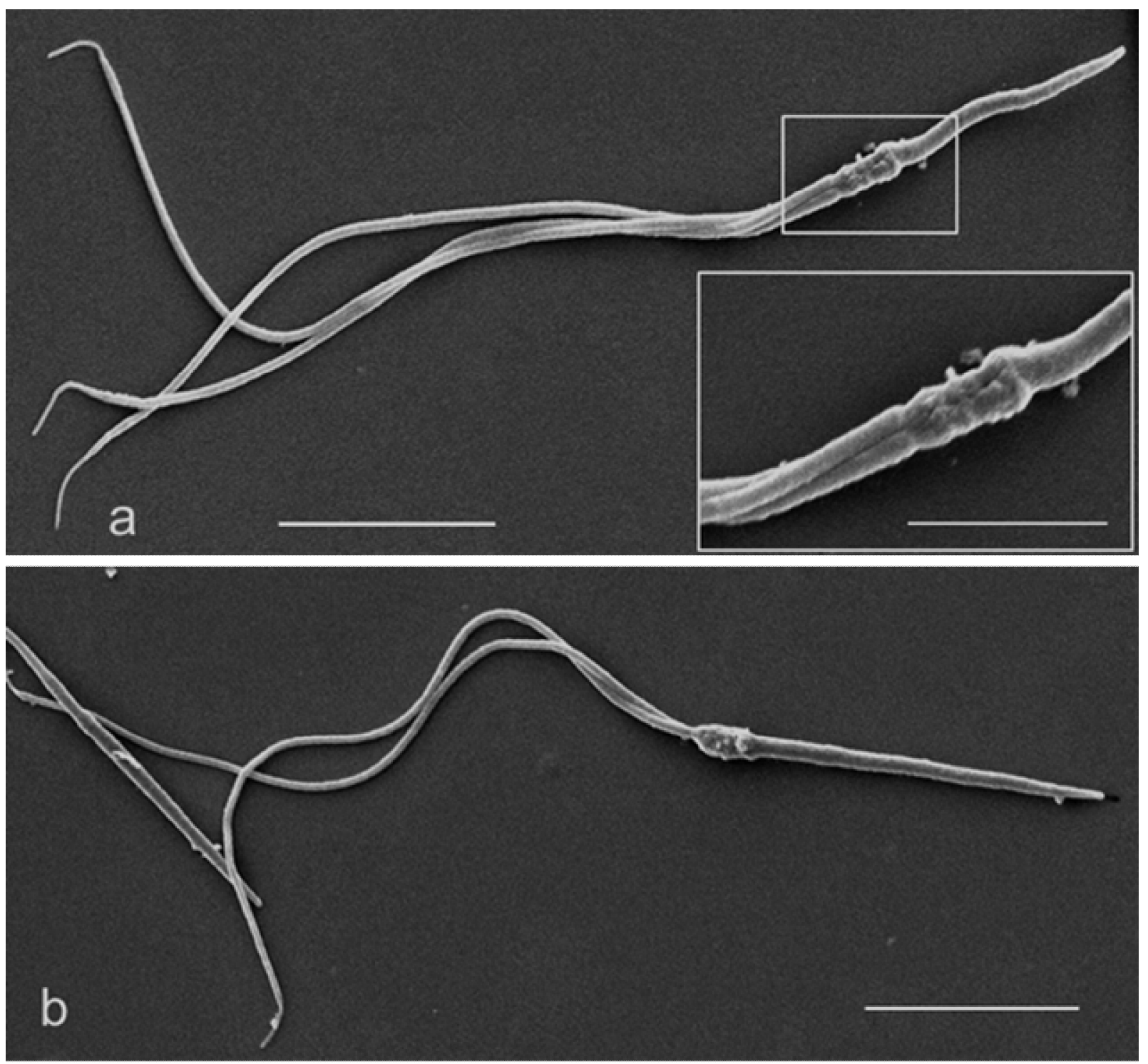

Fig. 2. Scanning electron micrographs showing (a) a normal-sized sperm with three tails and (b) a macrocephalic sperm with two tails. Note the spiralling of the proximal aspect of the principal piece in both defective cells. Inset: Magnification of the rectangle in Fig. 2a showing the 'common' midpiece (Mp) studded with mitochondria and the free emergence of the 3 principal pieces (arrow). Bar $=10 \mu \mathrm{m}$; inset bar $=5 \mu \mathrm{m}$. 

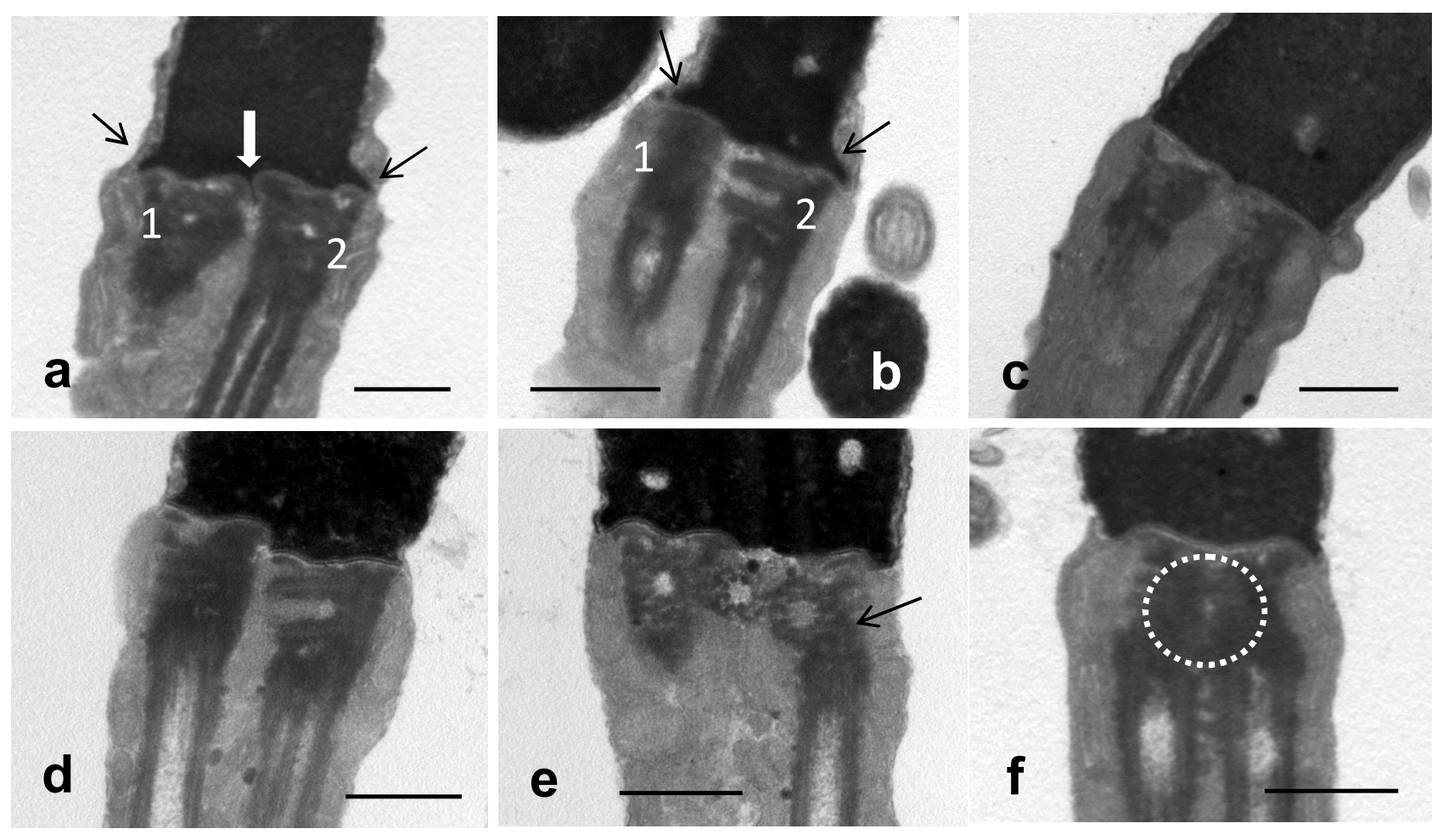

Fig. 3. Transmission electron micrographs of the head-base, neck and proximal midpiece of multiflagellate sperm. Figs. 3(a) and (b) illustrate sperm of normal head dimensions. Note the widened but peripherally attenuated nuclear base (black arrows) and the medial spine of karyoplasm (white arrow). The proximal centriolar pair (1 and 2) is similarly oriented in (a) but alternately oriented in (b) (see also Fig. 3d). Figs. 3 (c) to (f) represent macrocephalic multiflagellate sperm. The nuclear base appears flat (c), stepped (d) or scalloped (e) and does not display the lateral flaring observed in (a) and (b). In (e) note the close apposition of the triple proximal centrioles and the misalignment of the one set of proximal and distal centrioles (black arrow). In Fig. 3(f) only one proximal centriole (outlined) is obvious and shares contact with the two distal centrioles. Bar $=0.5 \mu \mathrm{m}$.

When viewed by TEM, longitudinal sections of the neck and midpiece of affected sperm revealed the presence of two or more centriolar complexes (Fig. 3). In biflagellate sperm with normal head dimensions the proximal aspect of the twin centriolar complexes were associated with duplicated elements of the connecting piece and, due to the limited space available at the base of the head, each attached abaxially to the nucleus via shallow, twin implantation fossae (Fig. 3c). In some cells the 
nuclear base was generally abnormally attenuated at the periphery, artificially widening the base to accommodate the multiple tails (Figs. 3a,b), while in others the nuclear base was stepped (Fig. 3d). In favourable sections, each implantation fossa resembled that of normal sperm, being composed of two shallow impressions in the nuclear base, each housing part of the capitellum and one of the poorly defined segmented columns that formed the connecting piece [10]. In some defective cells the fossae appeared shallow and the elements of the connecting piece appeared incomplete. Whether this was a true reflection of structural deficiencies or due to the plane of section could not be determined. The twin fossae were separated from each other by a conspicuous medial spine of karyoplasm. The two proximal centrioles were generally similarly oriented in respect of the nuclear base (Fig. 3a) although in some cells the orientation alternated, one centriole being observed in longitudinal profile and the other in transverse profile (Fig. 3b). In rare instances a single proximal centriole and two distal centrioles were observed. A distinct gap was generally present between the proximal centrioles and which was filled by homogeneous, moderately electron-dense material or in some instances by a mitochondrion emanating from the midpiece. The proximal and distal centrioles were generally linearly arranged but were occasionally misaligned, particularly when more than two centriolar complexes were present.

In macrocephalic multiflagellar sperm the head (nuclear) base was distinctly wider (average width $1.02 \pm 0.13 \mu \mathrm{m}$ ) than that of multiflagellar sperm of normal dimensions (average width $0.66 \pm$ $0.05 \mu \mathrm{m})$ and did not display the degree of peripheral attenuation typical of these cells. The larger heads comfortably accommodated the supernumerary centriolar complexes within the confines of the nuclear base. In instances where more than two proximal centrioles were present, the base of the nucleus presented a series of shallow depressions and the centrioles were compacted, with little 
space between them (Figs. 3a,b). All other structural features were similar to those described for normal-sized multiflagellar sperm. When three or more centriolar complexes were present they were circularly arranged beneath the nuclear base (Figs. 4a,b).

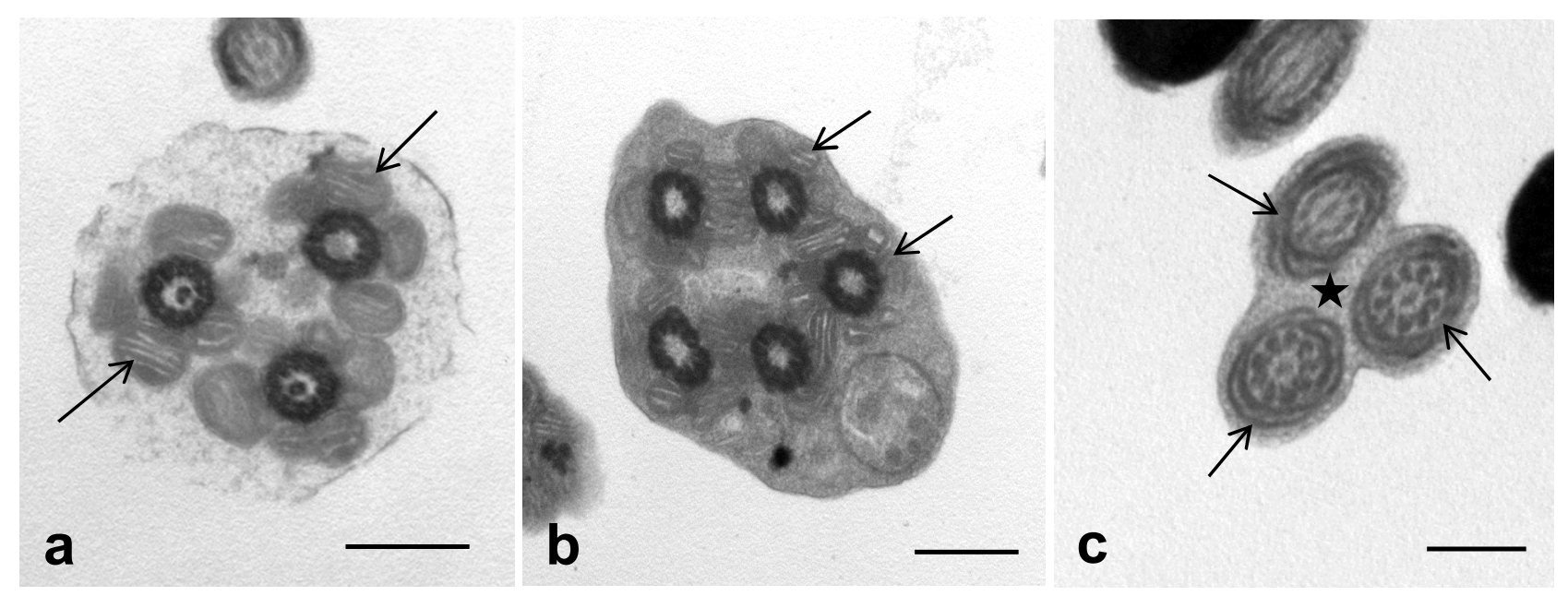

Fig. 4. Transverse sections of the midpiece of multiflagellate sperm displaying three (a) and five (b) distal centrioles respectively. The centrioles are circularly arranged and are surrounded by their respective mitochondrial sheaths (arrows). Fig. 4(c) represents a transverse section through the proximal principal piece of a cell with triple tails bound together by the plasmalemma. Each tail demonstrates the typical fibrous sheath (arrows) and axoneme characteristic of this region. Flocculant material (asterisk) occupies the space between the supernumerary tails. $\mathrm{Bar}=0.5 \mu \mathrm{m}$.

In all multiflagellar sperm the duplicated distal centrioles were arranged in parallel and extended the full length of the midpiece (from the neck region to the annulus). Each centriole was closely invested by its respective mitochondrial sheath although only a single row of mitochondria was present between adjacent centriolar complexes. This phenomenon was obvious in both longitudinal (Fig. 5) and transverse sections (Figs. 4a,b) of the midpiece. The impression on SEM that a double midpiece was present in some biflagellate sperm was not confirmed by TEM. The transition between the midpiece and principal piece was marked by a poorly developed but distinct annulus. The individual 
principal pieces of multiple flagella emerged freely from the collective midpiece, although in some cells the proximal part of the respective principal pieces was bound together by the plasmalemma

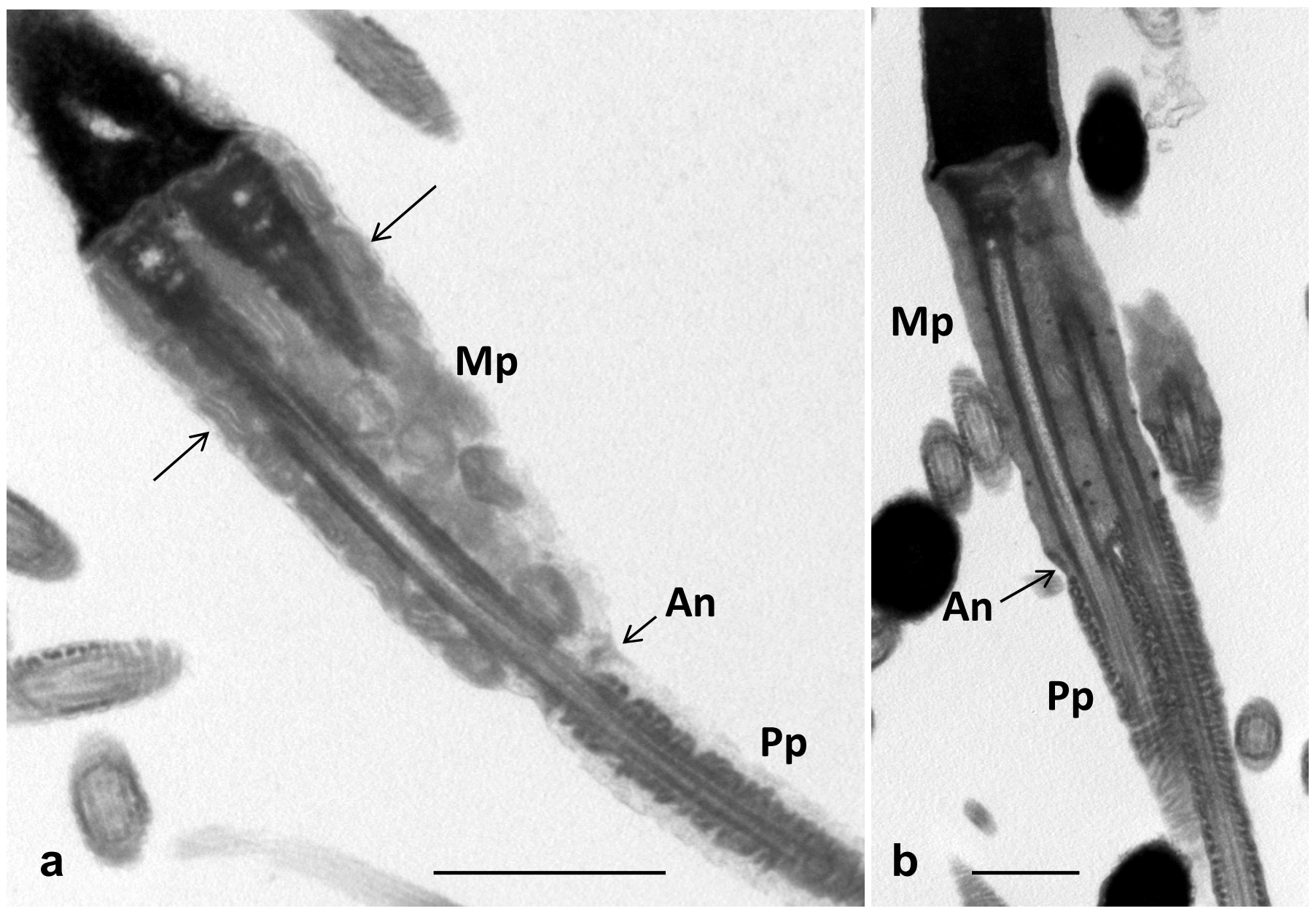

Fig. 5. Longitudinal sections of biflagellate sperm showing the unitary midpiece (Mp) containing the twin centriolar complexes surrounded by their respective mitochondrial sheaths (arrows). In (a), due to the plane of section, only a single principal piece $(\mathrm{Pp})$ appears to be present. The annulus (An) is indistinct. In (b) the twin principal pieces (Pp) emerge independently from the midpiece although they are closely apposed. Bar $=1 \mu \mathrm{m}$.

(Fig. 4c). The structural components of the midpiece and principal piece (axoneme, mitochondrial sheath, annulus and ribbed sheath) appeared normal. 
Due to the paucity of the defect and the difficulty in obtaining favourable sections, coupled with the similarity in morphological features between secondary spermatocytes and round spermatids, it was not possible to determine at what stage of spermatogenesis centriolar duplication was initiated. In
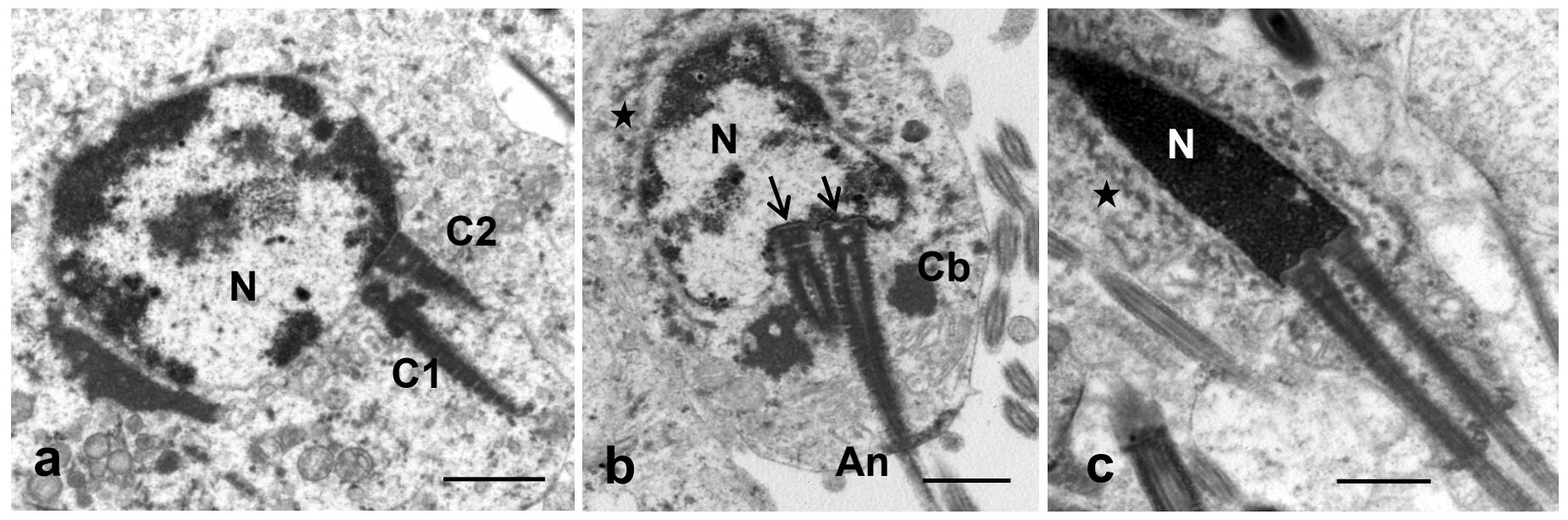

Fig. 6. Various stages in the formation of biflagellate sperm in the testis. (a) A round spermatid demonstrating the close proximity of twin centriolar complexes (C1 and $\mathrm{C} 2)$ to the nuclear membrane but without obvious attachment. (b) Spermatid with an irregular shaped nucleus showing attachment of the twin centriolar complexes (arrows). Note the presence of the circular manchette (asterisks). (c) An elongated spermatid illustrating the typical positioning of the twin flagella relative to the nucleus. The nucleus contains coarse chromatin granules and the circular manchette (asterisks) is still evident. Nucleus (N), Chromatoid body (Cb), Annulus (An). Bar $=1 \mu \mathrm{m}$.

the testis, the biflagellate form of the defect was again most obvious and was characterised by the appearance of twin centriolar complexes close to the nuclear membrane in round spermatids (Fig. 6a). Contact between the proximal centrioles and the nucleolemma occurred during this stage of spermiogenesis but was more obvious in spermatids displaying irregular-shaped nuclei (Fig. 6b). At this stage the twin implantation fossae and attendant structures around the proximal centriole (basal plate, forming capitellum, segmented columns) displayed the characteristic features of mature sperm. 
The irregular-shaped nucleus was surrounded by elements of the circular manchette and the chromatoid body was often closely associated with the centriolar complexes. The posterior aspect of the distal centriole was surrounded by a distinct annulus at its point of attachment to the plasmalemma (Fig. 6b). From this point the flagellar axoneme emerged and extended freely between the elements of the seminiferous epithelium. Early elongated spermatids (displaying coarse chromatin granules and the circular manchette) (Fig. 6c) and late elongated spermatids (with homogeneous, electron-dense chromatin and exhibiting the longitudinal manchette) displayed the morphological features typical of mature biflagellate sperm.

\section{Discussion}

Although multiflagellate sperm have been identified in a range of mammalian species, contrasting information has been presented on the occurrence $[18,19]$ and incidence of this defect. Some reports suggest that, when present, the incidence of this anomaly is high [20,26,27], while Pinart et al [23] regard it as a rare defect with a $1 \%$ incidence being considered normal. The literature on avian sperm defects is relatively silent on the occurrence, let alone incidence, of multiflagellate sperm in birds, probably due to the scarcity of the defect in this vertebrate group. The finding in the present study that multiflagellate sperm comprised on average only $1 \%$ of the total cell count reinforces this position.

In humans, multiple tails occur most frequently in association with macrocephalic sperm [46], with Escalier [27] reporting an average of 3.6 flagella originating from each sperm head. A link between macrocephalic sperm and multiple flagella was also apparent in the emus studied where $0.6 \%$ of cells with multiple tails were associated with giant heads. In the Houbara bustard macrocephalic sperm are 
reported to occasionally be associated with multiple tails [40]. Although macrocephalic sperm have been reported in other avian species [6,32,47-49], no mention is made of multiple tails or other defects associated with this anomaly. A more careful assessment of avian sperm defects may clarify this situation.

The structural features of multiflagellate sperm in the emu, particularly of the biflagellate form, were similar to those described in the drake [44]. The main features identified in both species were the duplicated (and apparently structurally normal) centriolar complexes occupying a common midpiece, peripheral widening of the nuclear base, duplicated shallow implantation fossae and sharing of mitochondria between adjacent centriolar complexes. The presence of a single proximal centriole associated with two distal centrioles was occasionally observed in both bird species. In common with defective drake sperm, the proximal part of the supernumerary principal pieces in the emu were sometimes contained within a common plasmalemma. However, the structurally deformed mitochondria of the midpiece and defective annulus reported in biflagellate drake sperm [44], were not observed in the emu. The poorly developed annulus of emu sperm may account for this discrepancy.

Multiflagellate sperm in birds appear to share the basic structural features described in multiflagellate mammalian sperm [19,26,27]. However, certain disparities are apparent. The most obvious difference involves the accommodation of the supernumerary centriolar complexes beneath the nuclear base. In mammalian sperm the broad nuclear base provides enough space for the implantation of multiple tails, albeit abaxially [19]. In emu and drake [44] sperm of normal dimensions the narrow, cylindrical head base displays tenuous peripheral widening in order to accommodate the additional tails. This observation points to the pliability of the nuclear membrane 
and nuclear base during spermiogenesis in birds. Another noticeable difference is the deep implantation fossae associated with mammalian sperm in contrast to the shallow fossae typical of the emu and drake. In multiple tailed human sperm, the axonemal complexes often appear to be defective [26,27]. However, this was not observed in the emu.

Consensus of opinion would seem to suggest that multiflagellate sperm have a dual origin in mammals. On the one hand, disruption of meiotic division during spermatogenesis and the resultant production of diploid and polyploid cells (essentially the fusion of two or more potential sperm) would account for the high proportion of macrocephalic sperm with multiple tails reported in various animals and man $[19,20,29-31,46]$. On the other hand, as suggested by several authors $[27,31,50]$, abnormal cycles of centriole formation would similarly result in multiflagellate sperm. A similar situation is apparent in the emu as reflected in the proportion of macrocephalic multiflagellate sperm $(0.6 \%)$ to multiflagellate sperm of normal dimensions $(0.4 \%)$. Examination of testicular material suggests that multiflagellate sperm (particularly the biflagellate form) of normal dimensions are the result of duplication of the centriolar complex in the emu. However, convincing structural evidence of incomplete or disrupted meiotic division leading to large cells with multiple flagella was not observed. In birds, therefore, a distinction should be made between sperm of normal dimensions with multiple tails (classified as a tail defect) resulting from abnormal centriolar duplication and macrocephalic sperm with multiple tails (classified as multiple defect - macrocephalic and multiflagellate) formed through incomplete cytokinesis [9].

A high percentage of multiple tails in man and bulls has been reported to adversely affect motility and fertility $[19,20,26,27]$. However the low incidence of this defect in mammals would suggest that it has little influence on fertility. Multiflagellate sperm constituted only $1 \%$ of the total cell count in 
the emus studied and consequently would have had little effect on semen quality and fertility in this species. However, the occurrence of this defect indicates a serious disturbance of normal spermiogenesis which, in instances where the incidence is high, could have a marked influence on fertility in avian species

\section{Acknowledgements}

The authors thank Mr. P. Bezuidenhout, Chairman of the Eastern Cape Emu Breeders Association and Mrs. P. Rough for making material available and the Grahamstown Ostrich Abattoir and Emu Ranch, Rustenburg, Northwest Province, South Africa for use of their facilities.

\section{References}

[1] Malecki IA, Rybnik PK, Martin GB. Artificial insemination technology for ratites: a review. Aust J Exp Agr 2008;48:1284-92.

[2] Ciereszko A, Rybnik PK, Horbańczuk JO, Dietrich GJ, Deas A, Słowińska M. 2010. Biochemical characterization and sperm motility parameters of ostrich (Struthio camelus) semen. Anim Reprod Sci 2010;122:222-8.

[3] Bonato M, Rybnik PK, Malecki IA, Cornwallis CK, Cloete SWP. Twice daily collection yields greater semen output and does not affect male libido in the ostrich. Anim Reprod Sci 2011; 123:25864. 
[4] Sood S, Malecki IA, Tawang A, Martin GB. 2011. Response of spermatozoa from the emu (Dromaius novaehollandiae) to rapid cooling, hyperosmotic conditions and dimethylacetamide (DMA). Anim Reprod Sci 2011;129:89-95.

[5] Bertschinger HJ, Burger WP, Soley JT, de Lange JH. Semen collection and evaluation of the male ostrich. Proc Biennial Congr S Afr Vet Assoc, Grahamstown, 1992, pp. 154-8.

[6] Malecki IA, Cummins JM, Martin GB, Lindsay DR. Effect of collection frequency on semen quality and the frequency of abnormal forms of spermatozoa in the emu. P Aus S Anim 1998;22:406.

[7] Soley JT, Els HJ. The ultrastructure of retained cytoplasmic droplets in ostrich spermatozoa. Proc Microsc Soc South Afr 1993;23:58.

[8] Soley JT, Bertschinger HJ, Els HJ, Burger WP. The morphology and incidence of retained cytoplasmic droplets in ostrich spermatozoa. In: Deeming DC (Ed.), Improving our Understanding of Ratites in a Farming Environment. Ratite Conference, Manchester, UK, 1996, pp. 16-8.

[9] Du Plessis L, Soley JT. Incidence, structure and morphological classification of abnormal sperm in the emu (Dromaius novaehollandiae). Theriogenology 2011;75:589-601.

[10] Du Plessis L, Soley JT. Head-base bending and disjointed spermatozoa in the emu (Dromaius novaehollandiae): a morphological comparison of two closely related defects. Theriogenology 2011;76:1258-65. 
[11] Du Plessis L, Soley JT. Abaxial tail implantation in the emu, Dromaius novaehollandiae: morphological characteristics and origin of a rare avian sperm defect. Theriogenology 2012; in press.

[12] Hotchkiss RS, Brunner EK, Grenley P. Semen analysis of two hundred fertile men. Am J Med Sci 1938;196:362-84.

[13] MacLeod J, Gold RZ. The male factor in fertility and infertility, IV: sperm morphology in fertile and infertile marriage. Fertil Steril 1951;2:394-414.

[14] Zamboni Z. Sperm structure and its relevance to infertility: an electron microscopic study. Arch Pathol Lab Med 1992;116:325-44.

[15] Jørgensen N, Auger J, Giwercman A, Irvine DS, Jensen TK, Jouannet P, Keiding N, Le Bon C, Macdonald E, Pekuri A-M, Scheike T, Simonsen M, Suominen J, Skakkebœk NE. Semen analysis performed by different laboratory teams: an intervariation study. Int J Androl 1997; 20:201-8.

[16] Cooper TG, Björndahl L, Vreeburg J, Nieschlag E. Semen analysis and external quality control schemes for semen analysis need global standardization. Int J Androl 2002;25:306-11.

[17] Arns MJ, Neck KF, Evans JW, Caceci T. Ultrastructural abnormalities in equine spermatozoa from a cryptorchid stallion. J Equine Vet Sci 1988;8:122-4.

[18] Brito LFC. Evaluation of stallion sperm morphology. Clin Tech Equine Prac 2007;6:249-64. 
[19] Barth AD, Oko RJ. Abnormal Morphology of Bovine Spermatozoa. Iowa State University Press, 1989.

[20] Kopp C, Sukura A, Tuunainen E, Gustavsson I, Parvinen M, Anderson M. Multinuclearmultiflagellar sperm defect in a bull - a new sterilizing sperm defect. Reprod Dom Anim 2007;42:208-13.

[21] Bonet S, Briz M. New data on aberrant spermatozoa in the ejaculate of Sus domesticus. Theriogenology 1991;35:725-30.

[22] Briz MD, Bonet S, Pinart E, Camps R. Sperm malformations throughout the boar epididymal duct. Anim Reprod Sci 1996;43:221-39.

[23] Pinart E, Camps R, Briz MD, Bonet S, Egozcue J. Unilateral spontaneous abdominal cryptorchidism: structural and ultrastructural study of sperm morphology. Anim Reprod Sci 1998;49:247-68.

[24] Oettlé EE, Soley JT. Sperm abnormalities in the dog: a light and electron microscopic study. Vet Med Rev 1988;59:28-70.

[25] Holstein AF. Morphologische studien an abnormen spermatiden und spermatozoon des menschen. Virchows Arch A 1975;367:93-112. 
[26] Nistal M, Paniagua R, Herruzo A. Multi-tailed spermatozoa in a case with asthenospermia and teratospermia. Virchows Arch B 1977;26:111-8.

[27] Escalier D. Human spermatozoa with large heads and multiple flagella: a quantitative ultrastructural study of 6 cases. Biol Cell 1983;48:65-74.

[28] Holstein AF, Roosen-Runge EC, Schirren C. Illustrated Pathology of Human Spermatogenesis. Grosse Verlag, 1988.

[29] Weissenberg R, Aviram A, Golan R, Lewin LM, Levron J, Madgar I, Dor J, Barkai G, Goldman B. Concurrent use of flow cytometry and fluorescence in-situ hybridization techniques for detecting faulty meiosis in a human sperm sample. Mol Human Reprod 1998;4:61-8.

[30] Perrin A, Morel F, Moy L, Colleu D, Amice V, De Braekeleer M. Study of aneuploidy in largeheaded, multiple-tailed spermatozoa: case report and review of the literature. Fertil Steril 2008;90:13-7.

[31] Lewis-Jones I, Aziz N, Seshadri S, Douglas A, Howard P. Sperm chromosomal abnormalities are linked to sperm morphologic deformities. Fertil Steril 2003;79:212-5.

[32] Wakely WJ, Kosin IL. A study of the morphology of the turkey spermatozoa with special reference to the seasonal prevalence of abnormal types. Am J Vet Res 1951;12:240-5. 
[33] Kamar GAR, Rizik MAA. Semen characteristics of two breeds of turkeys. J Reprod Fert 1972;29:317-25.

[34] Alkan S, Baran A, Özdas ÖB, Evecen M. Morphological defects in turkey semen. Turk J Vet Anim Sci 2002;26:1087-92.

[35] Umapathy G, Sontakke S, Reddy A, Ahmed S, Shivaji S. Semen characteristics of the captive Indian white-backed vulture. Biol Reprod 2005;73:1039-45.

[36] Tabatabaei S, Batavani RA, Talebi AR. Comparison of semen quality in Indigenous and Ross broiler breeder roosters. J Anim Vet Adv 2009;8:90-3.

[37] Gee GF, Temple SA. Artificial insemination for breeding non-domestic birds. Sym Zool S $1978 ; 43: 51-72$.

[38] Gee GF, Bertschinger H, Donoghue AM, Blanco J, Soley JT. Reproduction in nondomestic birds: Physiology, semen collection, artificial insemination and cryopreservation. Avian Poult Biol Rev 2004;15:47-101.

[39] Stelzer G, Crosta L, Bürkle M, Krautwald-Junghanns M-E. Attempted semen collection using the massage technique and semen analysis in various psittacine species. J Avian Med Surg 2005;19:7-13. 
[40] Lindsay C, Staines HJ, McCormick P, McCullum C, Choulani F, Wishart GJ. Variability in the size of the nucleus in spermatozoa from the Houbara bustards, Chlamydotis undulate undulate. J Reprod Fert 1999;117:307-13.

[41] Marvan F, Rob O, Janeckova E. Classification of morphological abnormalities of gander sperm. Zuchthygiene 1981;16:176-83.

[42] Gee GF, Sexton TJ. Cryogenic preservation of semen from the Aleutian Canada goose (Branta Canadensis leucopareia). Zoo Biol 1990;9:361-71.

[43] Ferdinand A. Licht- und elektronenmikroskopische Untersuchungen zur Morphologie von Ganterspermatozoen. Dissertation zur erlangung des grades eines Doctor Medicinae Veterinariae. Hannover, 1992.

[44] Maretta M. Ultrastructure of double and multiple sperm tails in drakes. Vet Med Czech 1979;24:679-89.

[45] Nwakalor LN, Okeke GC, Njoku DC. Semen characteristics of the guinea fowl Numida meleagris meleagris. Theriogenology 1988;29:545-54.

[46] Revay T, Kopp C, Flyckt A, Taponen J, Ijäs R, Nagy S, Kovacs A, Rens W, Rath D, Hidas A, Taylor JF, Andersson M. Diploid spermatozoa caused by failure of the second meiotic division in a 
bull. Theriogenology 2010;73:421-28.

[47] Penfold LM, Wildt DE, Herzog TL, Lynch W, Ware L, Derrickson SE, Monfort SL. Seasonal patterns of LH, testosterone and semen quality in the Northern pintail duck (Anas acuta). Reprod Fertil Dev 2000;12:229-35.

[48] Klimowicz M, Łukaszewicz E, Dubiel A. Effect of collection frequency on quantitative and qualitative characteristics of pigeon (Columba livia) semen. Brit Poultry Sci 2005;46:361-5.

[49] Chelmońska B, Jerysz A, Łukaszewicz E, Kowalczyk A, Malecki I. Semen collection from Japanese quail (Coturnix japonica) using a teaser female. Turk J Vet Anim Sci 2008;32:19-24.

[50] Bartoov B, Eltes F, Weissenberg R, Lunenfeld B. Morphological characteristics of abnormal human spermatozoa using transmission electron microscopy. Arch Andrology 1980;5:305-22. 